\title{
ESTRATÉGIA DE ANÁLISE DA QUALIDADE DE MADEIRA DE Eucalyptus sp. PARA PRODUÇÃO DE CELULOSE ${ }^{1}$
}

\author{
José Lívio Gomide², Humberto Fantuzzi Neto ${ }^{3}$, Hélio Garcia Leite ${ }^{4}$
}

\begin{abstract}
RESUMO - Estudou-se uma nova técnica laboratorial de análise de qualidade da madeira de Eucalyptus sp. para produção de polpa celulósica kraft. A técnica consiste em realizar, simultaneamente, quatro cozimentos de cavacos utilizando diferentes cargas de álcali ativo para estabelecer a característica de deslignificação da madeira. Pelo uso de equações de regressão, calcula-se o álcali ativo necessário para obter o número kappa desejado, o rendimento, a viscosidade e álcali consumido para o kappa objetivo. Os estudos estatísticos evidenciaram que uma única batelada de quatro cozimentos é suficiente, não sendo necessário realizar repetições.
\end{abstract}

Palavras-chave: Eucalyptus sp., qualidade da madeira, celulose kraft, seleção de clones.

\section{A LABORATORY TECHNIQUE TO ESTABLISH EUCalyptus sp. WOOD QUALITY FOR KRAFT PULP PRODUCTION}

\begin{abstract}
A new laboratory technique was tested to determine Eucalyptus sp. wood quality for kraft pulp production. This technique consists in performing four cooks simultaneously using different active alkali charges to establish the wood delignification characteristics. Based on regression equation it is possible to determine the alkali charge necessary to obtain a desired kappa number and the yield, viscosity and the alkali consumed for the target kappa. Statistical analysis demonstrated that a set of four cooks without replication is sufficient to determine the wood quality characteristics.
\end{abstract}

Key words: Eucalyptus sp., wood quality, kraft pulp, clone selection

\section{INTRODUÇÃO}

Um fator importante na competitividade da indústria de celulose é a seleção de árvores para formação de florestas homogêneas que possibilitem a produção de polpa celulósica com alto rendimento industrial, baixo custo e elevada qualidade tecnológica. No Brasil, os programas de melhoramento florestal das empresas nacionais de celulose e papel destacam-se no cenário mundial pelos excelentes resultados alcançados na área de clonagem de Eucalyptus sp. Resultados de levantamentos realizados pela Sociedade Brasileira de Silvicultura, em 2002, indicaram que as indústrias nacionais já alcançaram o invejável patamar de $60 \mathrm{~m}^{3} /$ ha/ano como incremento médio anual de suas florestas clonais de Eucalyptus sp.

Os estudos de clonagem de Eucalyptus sp. para estabelecimento de florestas com a qualidade requerida pelas empresas requerem análises e testes de milhares de clones potenciais. A seleção de clones inicia-se com os aspectos silviculturais, abrangendo características de enraizamento, resistência a doenças, adaptação aos fatores edafoclimáticos, forma do fuste e, de fundamental importância, as características de crescimento e, conseqüentemente, a produtividade de

\footnotetext{
${ }^{1}$ Recebido para publicação em 07.7.2003 e aceito para publicação em 08.6.2004.

${ }^{2}$ Professor Titular da Universidade Federal de Viçosa, no Departamento de Engenharia Florestal - Viçosa, MG. ${ }^{3}$ Estudante de Doutorado - Universidade Federal de Viçosa, Departamento de Engenharia Florestal - Viçosa, MG. ${ }^{4}$ Professor Adjunto da Universidade Federal de Viçosa, no Departamento de Engenharia Florestal - Viçosa, MG.
} 
madeira. Depois de uma seleção preliminar, pelas características silviculturais, é indispensável a realização de análises tecnológicas que possibilitem a determinação de critérios de qualidade da madeira de, ainda, milhares de clones remanescentes, para programas de melhoramento florestal de grande envergadura. Apenas depois desses estudos silviculturais e tecnológicos é que são selecionados os clones para multiplicação e formação de florestas homogêneas que irão apresentar, após um período de rotação de cinco a sete anos, alta produtividade florestal e, também, alta qualidade tecnológica para produção de polpa celulósica.

Os estudos de caracterização de qualidade da madeira para produção de polpa celulósica envolvem a constituição química da madeira, suas características estruturais anatômicas e também, de grande importância, as características tecnológicas da transformação da madeira em polpa celulósica. Estudos exaustivos de todas as características tecnológicas dos clones em fase de seleção são extremamente dispendiosos e requerem tempo excessivamente longo, inviabilizando a realização completa desses estudos. Madeira de um único clone poderia representar um custo de alguns milhares de dólares para ser completamente caracterizada nas suas propriedades físicas, químicas e tecnológicas para produção de polpa celulósica, conforme demonstrado por Downes et al. (1997). Entretanto, apesar de dispendiosa, a caracterização da madeira é de fundamental importância e terá que, inevitavelmente, ser realizada. Normalmente, a solução desses problemas consiste na realização de apenas algumas das análises, consideradas indispensáveis, capazes de fornecer os subsídios necessários para uma seleção tecnicamente consciente. Normalmente, numa primeira etapa de seleção de clones por critérios tecnológicos de qualidade de madeira são recomendados os estudos de densidade básica, de constituição química fundamental da madeira e, inevitavelmente, de sua transformação em polpa celulósica.

Mesmo adotando critérios de minimização das análises indispensáveis para caracterização tecnológica, a realização de vários cozimentos laboratoriais de uma mesma amostra para produção de celulose pode tornar-se dispendiosa e demandar tempo excessivo. Uma das principais dificuldades é, portanto, a realização de vários cozimentos laboratoriais para estabelecer, com segurança estatística, a confiabilidade dos resultados.
Levantamento bibliográfico realizado para o desenvolvimento deste estudo demonstrou que a literatura especializada é extremamente escassa em estudos de investigação sobre o número de repetições necessárias de cozimentos laboratoriais para estabelecimentos de características tecnológicas de polpação kraft da madeira de Eucalyptus sp. Um estudo sobre esse assunto foi publicado por Flores et al. (2000), que informaram ser muito comum, nesse tipo de cozimento, ocorrerem erros metodológicos muito expressivos que interferem na significância dos resultados. Por causa dos erros metodológicos e das variabilidades das propriedades da celulose e do processo de conversão da madeira em celulose, esses autores concluíram ser necessária a realização de quatro a seis repetições de um mesmo cozimento para obtenção de resultados estatisticamente confiáveis. Entretanto, a realização de cozimentos laboratoriais com quatro a seis repetições poderá tornarse economicamente inviável num programa de melhoramento florestal de grande envergadura.

O presente estudo foi desenvolvido visando estabelecer uma nova metodologia, baseada num número mínimo de cozimentos, que possibilitasse, com segurança estatística, determinar a curva de deslignificação, pelo processo kraft, de madeiras de Eucalyptus spp. Essa metodologia visa também, utilizando os mesmos cozimentos, determinar, para um número kappa preestabelecido, a carga de álcali necessária para produção de celulose, o rendimento do processo, a viscosidade da polpa e o álcali consumido no processo de polpação.

\section{MATERIAL E MÉTODOS}

\section{Produção de cavacos}

Três árvores de um clone de Eucalyptus sp. apresentando DAP e alturas correspondentes às médias do povoamento foram seccionadas em cinco toretes de $50 \mathrm{~cm}$ de comprimento, retirados na base e a 25, 50, 75 e $100 \%$ da altura comercial. Como altura comercial da árvore foi considerado todo o tronco até a altura correspondente ao diâmetro de $6 \mathrm{~cm}$. As toras foram transformadas em cavacos por meio de um picador laboratorial de disco com três facas, dotado de duas peneiras classificadoras com aberturas de 39 x 39 mm e 6 x $6 \mathrm{~mm}$. Para alcançar alta homogeneidade, os cavacos foram reclassificados em peneira com abertura de 16 x 16 mm, sendo realizada também uma classificação 
manual para eliminar pedaços de casca, cavacos com defeitos de corte e madeira com nós. Depois de selecionados, os cavacos foram secados ao ar e armazenados em sacos de polietileno, para uniformização e conservação do teor de umidade. A amostragem dos cavacos para determinação do teor de umidade e realização dos cozimentos foi realizada pela técnica de quarteamento.

\section{Polpação kraft}

Os cozimentos kraft foram executados em digestor rotativo, com quatro reatores individuais de dois litros cada um, aquecido eletricamente e dotado de termômetro e manômetro, possibilitando a realização, numa única batelada, de quatro cozimentos simultaneamente. O tempo e a temperatura de cozimento foram monitorados por meio de controlador eletrônico acoplado a um computador, conforme mostrado na Figura 1. O sensor de temperatura foi um PT100, em contato direto com os cavacos e o licor de cozimento.

Na Figura 2 é apresentado o gráfico dos perfis de temperatura e de fator $\mathrm{H}$, determinados eletronicamente em cada batelada de cozimentos kraft.

Foram realizados, simultaneamente, quatro cozimentos em cada batelada, e a operação foi repetida cinco vezes, perfazendo 20 cozimentos. Em cada reator foi utilizada carga diferente de álcali ativo (14, 16, 18 e 20\%), expressa como $\mathrm{NaOH}$, para estabelecer a curva de deslignificação da madeira. Essas cargas de álcali foram selecionadas após a realização de cozimentos exploratórios, objetivando à obtenção de número kappa

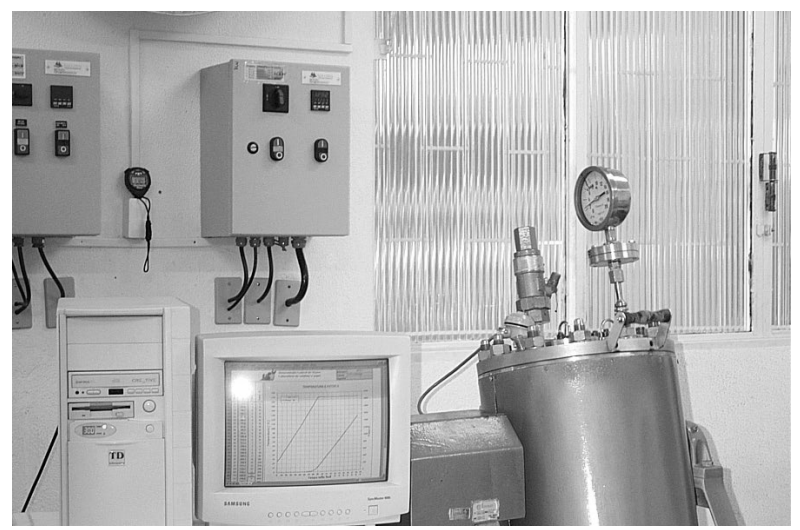

Figura 1 -Digestor Batch computadorizado, com quatro reatores. Figure 1 - Computerized batch digestor, four reactors.
PERFIL DE TEMPERATURA E FATOR H

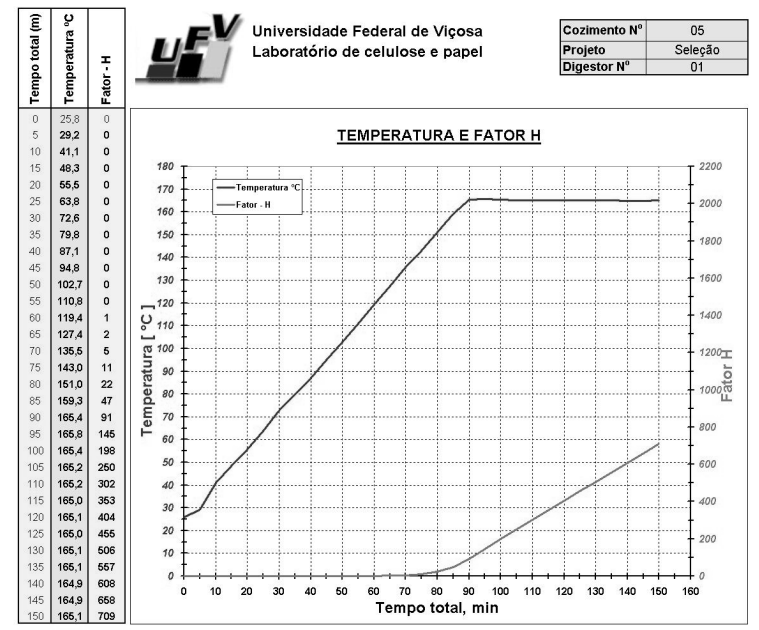

Figura 2 - Perfis de temperatura e de fator $\mathrm{H}$ do cozimento kraft.

Figure 2 - Temperature and H factor profiles for kraft cooks.

próximo a 18, na parte mediana da curva de deslignificação. Os outros parâmetros utilizados na polpação kraft foram os seguintes, mantidos constantes em todos os cozimentos: cavacos $=250$ g a.s., sulfidez $=25 \%$, relação licor $/$ madeira $=4 / 1$, temperatura máxima $=165^{\circ} \mathrm{C}$, tempo até a temperatura máxima $=90$ minutos e tempo à temperatura máxima $=60$ minutos. As operações de lavagem, de individualização das fibras dos cavacos cozidos e de depuração da polpa celulósica, foram realizadas conforme ilustrado na Figura 3.

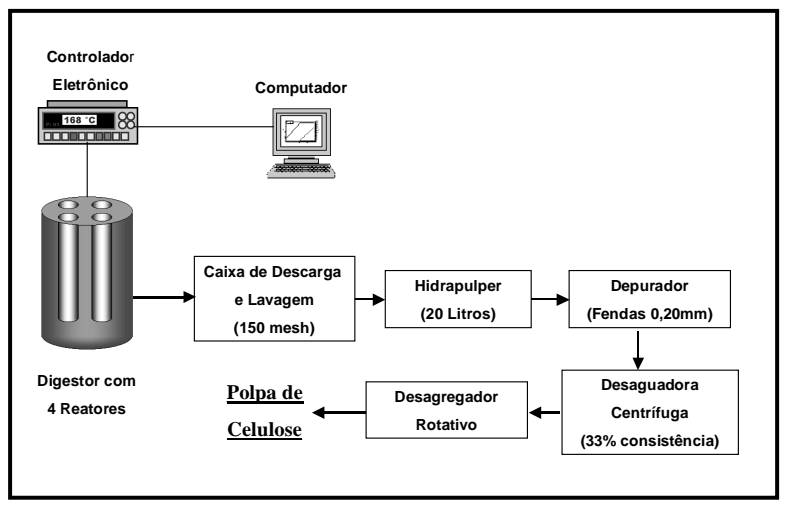

Figura 3 - Sistema batch computadorizado para produção e depuração da polpa celulósica.

Figure 3-Computerized batch digester system for pulp production and screening.

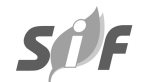

R. Árvore, Viçosa-MG, v.28, n.3, p. 443-450, 2004 
Após o cozimento, os cavacos foram descarregados dos reatores e lavados exaustivamente com água à temperatura ambiente, utilizando tela de aço inox de 150 mesh. A individualização das fibras foi realizada em “hidrapulper” laboratorial de 25 litros de capacidade, numa consistência de aproximadamente 0,6\%. A depuração da celulose foi realizada em depurador laboratorial Voith dotado de placa com fendas de 0,2 mm. A celulose, após lavagem, desfibramento e depuração, foi desaguada em centrífuga, a uma consistência de cerca de $30 \%$, sendo, depois da desagregação mecânica, armazenada em saco de polietileno para análises posteriores. Foram determinados o número kappa e a viscosidade das polpas celulósicas, utilizando-se normas Tappi; e as concentrações dos licores residuais foram determinadas segundo Grace (1989).

Foi feita a análise de regressão de cada batelada de quatro cozimentos realizados simultaneamente, estabelecendo-se equações para correlacionar número kappa, rendimento depurado, viscosidade e álcali consumido em função do álcali ativo aplicado. Pelo uso das equações, determinou-se o álcali ativo necessário para obter número kappa 18. Foram também determinados o rendimento do processo, a viscosidade das polpas e o álcali ativo consumido correspondentes ao número kappa 18.

\section{Análises estatísticas}

O objetivo básico deste estudo foi verificar se ocorriam diferenças estatisticamente significativas entre as cinco bateladas de cozimentos. A inexistência dessas diferenças demonstraria a possibilidade de utilização de apenas uma batelada de cozimentos para caracterização tecnológica da madeira. Estatisticamente, as hipóteses formuladas para a realização do estudo foram:

- $\mathrm{H}_{0}$ : é necessária a realização de pelo menos cinco repetições de um conjunto de quatro cozimentos simultâneos, utilizando-se, de cada vez, quatro cargas alcalinas diferentes para o estabelecimento da curva de deslignificação.

- $\mathrm{H}_{\mathrm{a}}$ : não $\mathrm{H}_{0}$.

Cada batelada de cozimentos, utilizando os quatro reatores e as cargas alcalinas de 14, 16, 18 e 20\%, constituiu, estatisticamente, uma amostra, perfazendo, portanto, um total de cinco amostras, codificadas como A1, A2, A3, A4 e A5. Para cada amostra foram determinadas quatro equações de regressão para expressar as variações do número kappa, do rendimento depurado, da viscosidade das polpas e do álcali ativo consumido, em razão do álcali ativo aplicado. Estabeleceram-se, portanto, para início das análises estatísticas, 20 equações de regressão, que foram comparadas entre si, para verificar se havia diferenças estatísticas entre elas.

\section{RESULTADOS E DISCUSSÃO}

Os resultados de todos os cozimentos, abrangendo números kappa, viscosidades, rendimentos, características dos licores residuais e os álcalis ativos consumidos durante os cozimentos, estão apresentados no Quadro 1.

Por meio de análise de regressão, determinou-se o modelo de regressão que melhor se ajustava aos resultados obtidos. Esse modelo foi o logaritmo, descrito pela equação ( $\left.\operatorname{Ln} \mathrm{Y}=\beta_{0}+\beta_{1} \mathrm{X}_{\mathrm{i}}+\mathrm{e}_{\mathrm{i}}\right)$, em que $\mathrm{Y}$ representa o número kappa, rendimento, viscosidade ou álcali consumido e $\mathrm{X}_{\mathrm{i}}$, as cargas de álcali ativo utilizadas. Tais equações estão apresentadas no Quadro 2.

Para verificar se havia diferenças estatísticas significativas entre os cozimentos, foram realizadas comparações entre os cozimentos e entre todas as possíveis combinações entre os cozimentos. Essas comparações, denominadas alternativas, encontramse no Quadro 3.

Inicialmente, procurou-se responder à seguinte pergunta: as equações de regressão determinadas para número kappa, rendimento depurado, viscosidade e álcali ativo consumido, abrangendo todos os possíveis conjuntos de alternativas (1-5, 6-15, 16-25 e 2630), eram estatisticamente iguais? Exemplificando melhor, para alternativas 6 a 15, por exemplo, pergunta-se: as equações geradas com qualquer combinação de duas amostras são estatisticamente iguais? Para responder a essa pergunta foi utilizado o modelo de regressão ( $\left.\operatorname{Ln} \mathrm{Y}=\beta_{0}+\beta_{1} \mathrm{X}_{\mathrm{i}}+\mathrm{e}_{\mathrm{i}}\right)$, e, para cada variável dependente $\mathrm{Y}$ ( $\mathrm{Y}$ = número kappa, rendimento depurado, viscosidade ou álcali ativo residual), ajustou-se o modelo completo em função da carga de álcali (X = álcali ativo), conforme mostrado na Equação (A).

Ln $\mathrm{Y}_{\mathrm{i}}=\beta_{1} \mathrm{D}_{1}+\beta_{2} \mathrm{D}_{2}+\ldots+\beta_{\mathrm{P}} \mathrm{D}_{\mathrm{P}}+\beta_{\mathrm{P}+1} \mathrm{D}_{1} \mathrm{X}_{\mathrm{i}}+\beta_{\mathrm{P}+2} \mathrm{D}_{2} \mathrm{X}_{\mathrm{i}}+$ $\ldots+\beta_{\mathrm{P}+\mathrm{P}} \mathrm{D}_{\mathrm{P}} \mathrm{X}_{\mathrm{i}}+\mathrm{ei} \quad$ Equação (A) em que:

Caso 1: $\mathrm{P}=5$ para dados de uma amostra (alternativas 1 a 5$)$. 
Quadro 1 - Cozimentos kraft dos cavacos de madeira de clone de Eucalyptus Table 1 - Kraft pulping of Eucalyptus wood

\begin{tabular}{|c|c|c|c|c|c|c|c|c|c|c|}
\hline \multirow{2}{*}{$\begin{array}{c}\text { Batelada } \\
\mathrm{N}^{\circ}\end{array}$} & \multirow{2}{*}{$\begin{array}{c}\text { Álcali } \\
\text { Ativo, \% }\end{array}$} & \multirow{2}{*}{$\begin{array}{l}\text { Número } \\
\text { kappa }\end{array}$} & \multirow{2}{*}{$\begin{array}{l}\text { Visc } \\
\text { cP }\end{array}$} & \multicolumn{3}{|c|}{ Rendimentos, \% } & \multicolumn{3}{|c|}{$\begin{array}{l}\text { Licor negro } \\
\text { residual }\end{array}$} & \multirow{2}{*}{$\begin{array}{c}\text { AA } \\
\text { consum. } \\
\%\end{array}$} \\
\hline & & & & Depurado & Rejeitos & Total & $\mathrm{pH}$ & $\mathrm{NaOH}$ & $\mathrm{Na}_{2} \mathrm{~S}$ & \\
\hline \multirow{4}{*}{1} & 14 & 26,6 & 66,3 & 48,8 & 0,1 & 48,9 & 13,5 & 1,2 & 3,1 & 12,3 \\
\hline & 16 & 20,5 & 49,9 & 47,4 & 0,0 & 47,4 & 13,8 & 2,7 & 3,9 & 13,4 \\
\hline & 18 & 18,1 & 38,9 & 46,1 & 0,0 & 46,1 & 14,0 & 5,0 & 4,1 & 14,4 \\
\hline & 20 & 16,5 & 31,6 & 45,2 & 0,0 & 45,2 & 14,1 & 7,0 & 5,3 & 15,1 \\
\hline \multirow{4}{*}{2} & 14 & 26,4 & 70,2 & 49,3 & 0,3 & 49,6 & 13,4 & 1,0 & 3,0 & 12,4 \\
\hline & 16 & 20,3 & 50,4 & 47,5 & 0,1 & 47,6 & 13,8 & 2,3 & 4,1 & 13,4 \\
\hline & 18 & 17,8 & 38,8 & 46,9 & 0,0 & 46,9 & 14,0 & 5,0 & 4,1 & 14,4 \\
\hline & 20 & 16,1 & 31,3 & 45,5 & 0,0 & 45,5 & 14,1 & 7,7 & 4,9 & 15,0 \\
\hline \multirow{4}{*}{3} & 14 & 25,7 & 74,9 & 49,3 & 0,1 & 49,4 & 12,7 & 1,0 & 3,6 & $\overline{12,2}$ \\
\hline & 16 & 19,8 & 48,4 & 47,5 & 0,0 & 47,5 & 13,0 & 2,9 & 4,1 & 13,2 \\
\hline & 18 & 17,5 & 40,4 & 46,7 & 0,0 & 46,7 & 13,1 & 5,1 & 4,7 & 14,1 \\
\hline & 20 & 16,4 & 31,9 & 45,7 & 0,0 & 45,7 & 13,2 & 6,9 & 5,3 & 15,1 \\
\hline \multirow{4}{*}{4} & 14 & 25,3 & 72,3 & 49,1 & 0,1 & 49,2 & 12,7 & 1,2 & 3,3 & 12,2 \\
\hline & 16 & 19,5 & 49,9 & 47,5 & 0,0 & 47,5 & 13,0 & 2,6 & 3,8 & 13,4 \\
\hline & 18 & 17,5 & 39,1 & 46,5 & 0,0 & 46,5 & 13,1 & 4,9 & 3,8 & 14,5 \\
\hline & 20 & 16,4 & 32,8 & 45,6 & 0,0 & 45,6 & 13,1 & 6,6 & 4,5 & 15,6 \\
\hline \multirow{4}{*}{5} & 14 & 26,7 & 70,5 & 49,0 & 0,3 & 49,3 & 12,6 & 0,9 & 3,4 & 12,3 \\
\hline & 16 & 19,6 & 48,9 & 48,1 & 0,0 & 48,1 & 13,0 & 3,1 & 3,9 & 13,2 \\
\hline & 18 & 17,8 & 38,8 & 46,1 & 0,0 & 46,1 & 13,1 & 4,5 & 4,5 & 14,4 \\
\hline & 20 & 16,8 & 32,2 & 45,5 & 0,0 & 45,5 & 13,2 & 6.9 & 5,0 & 15,2 \\
\hline
\end{tabular}

Quadro 2 - Modelos de regressão determinados para as características avaliadas

Table 2 - Regression models for pulp characteristics

\begin{tabular}{llc}
\hline Amostras & \multicolumn{1}{c}{ Equações de Regressão } & $\mathrm{R}^{2}$ \\
\hline 1 & Ln kappa $=4,3237-0,0779 \times$ AA \% & 0,94 \\
2 & Ln kappa $=4,3583-0,0808$ x AA \% & 0,94 \\
3 & Ln kappa $=4,2234-0,0736$ x AA \% & 0,92 \\
4 & Ln kappa $=4,1626-0,0704 \times$ AA \% & 0,91 \\
5 & Ln kappa $=4,2535-0.0743 \times$ AA \% & 0,86 \\
\hline 1 & Ln rend. depur. $=4,0661-0,0129 \times$ AA \% & 0,99 \\
2 & Ln rend. depur. $=4,0714-0,0127 \times$ AA \% & 0,97 \\
3 & Ln rend. depur. $=4,0639-0,0122 \times$ AA \% & 0,97 \\
4 & Ln rend. depur. $=4,0601-0,0121 \times$ AA \% & 0,98 \\
5 & Ln rend. depur. $=4,0785-0,0132 \times$ AA \% & 0,96 \\
\hline 1 & Ln viscosidade $=5,9059-0,1236 \times$ AA \% & 0,99 \\
2 & Ln viscosidade $=6,1003-0,1342 \times$ AA \% & 0,99 \\
3 & Ln viscosidade $=6,1694-0,1371 \times$ AA \% & 0,96 \\
4 & Ln viscosidade $=6,0597-0,1307 \times$ AA \% & 0,97 \\
5 & Ln viscosidade $=6,0139-0,1291 \times$ AA \% & 0,98 \\
\hline 1 & Ln AA consumido $=2,0343+0,0344 \times$ AA \% & 0,99 \\
2 & Ln AA consumido $=2,0866+0,0315 \times$ AA \% & 0,98 \\
3 & Ln AA consumido $=1,9994+0,0359 \times$ AA \% & 1,00 \\
4 & Ln AA consumido $=1,9439+0,0404 \times$ AA \% & 0,99 \\
5 & Ln AA consumido $=1,9952+0,0367 \times$ AA \% & 0,99 \\
\hline
\end{tabular}

Quadro 3 - Análises comparativas (alternativas) realizadas entre as equações estabelecidas para cada batelada de cozimentos (amostras)

Table 3-Comparative analyses (alternatives) performed for equations established for each cook batch (samples)

\begin{tabular}{ccccc}
\hline Alternativas & Amostras & & Alternativas & Amostras \\
\cline { 1 - 2 } \cline { 5 - 5 } 2 & A1 & & 16 & $\mathrm{~A} 1+\mathrm{A} 2+\mathrm{A} 3$ \\
3 & $\mathrm{~A} 2$ & & 17 & $\mathrm{~A} 1+\mathrm{A} 2+\mathrm{A} 4$ \\
4 & $\mathrm{~A} 3$ & & 18 & $\mathrm{~A} 1+\mathrm{A} 2+\mathrm{A} 5$ \\
5 & $\mathrm{~A} 4$ & & 19 & $\mathrm{~A} 1+\mathrm{A} 3+\mathrm{A} 4$ \\
6 & $\mathrm{~A} 5$ & & 20 & $\mathrm{~A} 1+\mathrm{A} 3+\mathrm{A} 5$ \\
7 & $\mathrm{~A} 1+\mathrm{A} 2$ & & 21 & $\mathrm{~A} 1+\mathrm{A} 4+\mathrm{A} 5$ \\
8 & $\mathrm{~A} 1+\mathrm{A} 4$ & 22 & $\mathrm{~A} 2+\mathrm{A} 3+\mathrm{A} 4$ \\
9 & $\mathrm{~A} 1+\mathrm{A} 5$ & 24 & $\mathrm{~A} 2+\mathrm{A} 3+\mathrm{A} 5$ \\
10 & $\mathrm{~A} 2+\mathrm{A} 3$ & 25 & $\mathrm{~A} 3+\mathrm{A} 4+\mathrm{A} 5$ \\
11 & $\mathrm{~A} 2+\mathrm{A} 4$ & 26 & $\mathrm{~A} 1+\mathrm{A} 2+\mathrm{A} 3+\mathrm{A} 4$ \\
12 & $\mathrm{~A} 2+\mathrm{A} 5$ & 27 & $\mathrm{~A} 1+\mathrm{A} 2+\mathrm{A} 3+\mathrm{A} 5$ \\
13 & $\mathrm{~A} 3+\mathrm{A} 4$ & 28 & $\mathrm{~A} 1+\mathrm{A} 2+\mathrm{A} 4+\mathrm{A} 5$ \\
14 & $\mathrm{~A} 3+\mathrm{A} 5$ & 29 & $\mathrm{~A} 1+\mathrm{A} 3+\mathrm{A} 4+\mathrm{A} 5$ \\
15 & $\mathrm{~A} 4+\mathrm{A} 5$ & 30 & $\mathrm{~A} 2+\mathrm{A} 3+\mathrm{A} 4+\mathrm{A} 5$ \\
\hline
\end{tabular}


Caso 2: $\mathrm{P}=10$ para dados de duas amostras (alternativas 6 a 15).

Caso 3: $P=10$ para dados de três amostras (alternativas 16 a 25).

Caso 4: $\mathrm{P}=5$ para dados de quatro amostras (alternativas 26 a 30).

Para cada caso, ajustaram-se o modelo completo (A) e o modelo reduzido mostrado na Equação (B).

$\operatorname{Ln} \mathrm{Y}_{\mathrm{i}}=\beta_{0}+\beta_{1} \mathrm{X}_{\mathrm{i}}+\mathrm{e}_{\mathrm{i}}$ Equação (B)

Em seguida, computou-se a estatística $\mathrm{F}\left(\mathrm{H}_{0}\right)$, dada por:

$$
\mathrm{F}\left(\mathrm{H}_{0}\right)=\frac{\mathrm{R}\left(\mathrm{H}_{0}\right) / \mathrm{GL} \cdot \mathrm{R}\left(\mathrm{H}_{0}\right)}{\text { SQRes } / \mathrm{GL} \cdot \text { Resíduo }}
$$

em que:

$\mathrm{R}\left(\mathrm{H}_{0}\right)=\mathrm{SQ}$ Parâmetros (A) - SQ Parâmetros (B);

SQ Res = SQ Total - SQ Parâmetros (A); e

$\mathrm{F}\left(\mathrm{H}_{0}\right)$, sob normalidade, segue distribuição F central, com graus de liberdade GL. $\left(\mathrm{H}_{0}\right)$ e GL.Resíduo.

A hipótese avaliada para cada equação por essa estatística $\mathrm{F}\left(\mathrm{H}_{0}\right)$ foi: $\mathrm{H}_{0}=$ as equações obtidas foram estatisticamente iguais e $\mathrm{H}_{\mathrm{a}}=$ não $\mathrm{H}_{0}$. Os graus de liberdade das equações estão apresentados no Quadro 4.

Esta análise foi necessária para verificar se:

a) As equações obtidas com uma amostra casualmente selecionada eram iguais às das outras amostras.

b) As equações obtidas com quaisquer combinações de duas amostras eram iguais.

c) As equações obtidas com quaisquer combinações de três amostras eram iguais.

Quadro 4 - Graus de liberdade dos testes de identidade de modelos

Table 4 - Degree of freedom for model identity tests

\begin{tabular}{lrrrr}
\hline \multirow{2}{*}{ FV } & \multicolumn{4}{c}{ Graus de liberdade } \\
\cline { 2 - 5 } & Caso 1 & Caso 2 & Caso 3 & Caso 4 \\
\hline Par. (A) & 10 & 20 & 20 & 10 \\
Par. (B) & 2 & 2 & 2 & 2 \\
R $\left(\mathrm{H}_{0}\right)$ & 8 & 18 & 18 & 8 \\
Resíduo & 10 & 60 & 100 & 70 \\
Total & 20 & 80 & 120 & 80 \\
\hline
\end{tabular}

d) As equações obtidas com quaisquer combinações de quatro amostras eram iguais.

Se todas essas possibilidades (a, b, c, d) fossem estatisticamente comprovadas, poder-se-ia concluir que as equações para número kappa, rendimento, viscosidade e álcali consumido, obtidas com cinco bateladas de cozimentos, eram estatisticamente iguais entre si. Para verificar a existência dessas igualdades estatísticas, foi realizada a análise de variância das regressões do número kappa em função do álcali ativo aplicado (Quadro 5).

Em todas as equações analisadas, o F calculado foi menor que o F tabelado, provando a igualdade das regressões correspondentes a cada amostra estatística (batelada de cozimento), em todas as combinações possíveis. Esse mesmo teste estatístico foi aplicado com relação a viscosidade, rendimento e álcali ativo consumido, tendo também o F calculado sido sempre menor que o $\mathrm{F}$ tabelado, provando a identidade entre as regressões individuais e em todas as combinações possíveis.

O teste estatístico foi conclusivo, demonstrando a igualdade estatística entre as cinco bateladas de cozimentos. Isso significa que apenas uma equação de regressão obtida para quatro cozimentos com cargas alcalinas distintas é suficiente para o estabelecimento de curvas de regressões dos parâmetros avaliados neste estudo, ou seja, apenas uma batelada de quatro cozimentos é suficiente para estabelecer as características tecnológicas da madeira, sem necessidade de repetição dos cozimentos.

\section{Resultados da técnica proposta}

Para demonstrar a técnica proposta neste estudo, será utilizada apenas a primeira repetição das cinco bateladas (Quadro 1). Na Figura 4 estão apresentados os gráficos obtidos e as respectivas equações estabelecidas para determinar, matematicamente, a carga de álcali necessária à obtenção de número kappa 18, bem como o rendimento, a viscosidade e o álcali consumido, correspondentes a esse nível de kappa.

Pelo uso das equações, foram determinadas as seguintes características tecnológicas da madeira de Eucalyptus sp. utilizada neste estudo:

- Álcali ativo necessário para kappa 18 = 18,4\%.

- Rendimento correspondente a kappa $18=46,0 \%$. 
Quadro 5 - Análise de variância das regressões de número kappa em função do álcali ativo aplicado Table 5 - Analysis of variance of kappa number regressions as function of the active alkali applied.

\begin{tabular}{|c|c|c|c|c|c|c|c|c|c|c|c|c|c|c|c|c|}
\hline \multirow[b]{2}{*}{ FV } & \multicolumn{3}{|c|}{ Amostras individuais } & \multicolumn{5}{|c|}{ Combinação de 2 amostras } & \multicolumn{4}{|c|}{ Combinação de 3 amostras } & \multicolumn{4}{|c|}{ Combinação de 4 amostras } \\
\hline & GL & SQ & $\mathrm{QM}$ & $\mathrm{F}$ & GL & SQ & $\mathrm{QM}$ & $\mathrm{F}$ & GL & SQ & $\mathrm{QM}$ & $\mathrm{F}$ & GL & SQ & $\mathrm{QM}$ & $\mathrm{F}$ \\
\hline $\operatorname{Par}(\mathrm{A})$ & 10 & 178,5130 & & & 20 & 714.0410 & & & 20 & 1071.0560 & & & 10 & 714.0355 & & \\
\hline $\operatorname{Par}(\mathrm{B})$ & 2 & 178,5086 & & & 2 & 714.0347 & & & 2 & 1071.0517 & & & 2 & 714.0344 & & \\
\hline $\mathrm{R}(\mathrm{Ho})$ & 8 & 0,0044 & 0,0006 & 0,11 & 18 & 0.0063 & 0,0004 & 0.10 & 18 & 0.0043 & 0.0002 & 0.07 & 8 & 0.0011 & 0.0001 & 0.04 \\
\hline Resíduo & 10 & 0,0520 & 0,0052 & & 60 & 0.2191 & 0,0037 & & 100 & 0.3341 & 0.0033 & & 70 & 0.2245 & 0.0032 & \\
\hline Total & 20 & 178,5650 & & & 80 & 714.2601 & & & 120 & 1071.3901 & & & 80 & 714.2600 & & \\
\hline
\end{tabular}
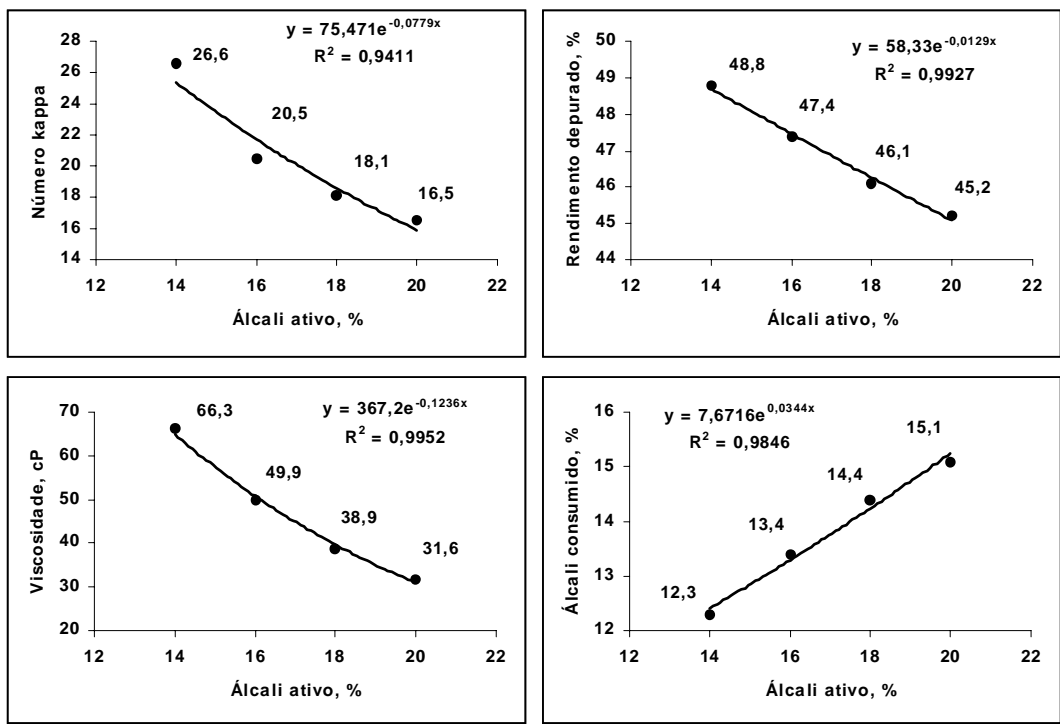

Figura 4 - Características de deslignificação da madeira de Eucalyptus.

Figure 4 - Delignification characteristics of Eucalyptus wood.

- Viscosidade correspondente a kappa $18=37,8 \mathrm{cP}$.

- Álcali ativo consumido correspondente a kappa $18=14,4 \%$.

Existem programas que selecionam clones com base no custo simulado de produção de polpa (FONSECA et al., 1996). Nesses programas, as informações necessárias para a classificação dos clones podem ser obtidas pela técnica proposta neste trabalho.

\section{CONCLUSÃO}

A metodologia proposta para avaliação tecnológica da qualidade de madeira de clones de Eucalyptus sp. pode ser feita com apenas uma batelada de quatro cozimentos com diferentes cargas de álcali ativo. Podese determinar a curva de deslignificação da madeira e, utilizando equação de regressão, calcular a carga de álcali necessária para obter o número kappa desejado (neste estudo foi selecionado o número kappa 18). Empregando-se equações de regressão, é possível calcular a viscosidade da polpa, o rendimento do processo de polpação e o álcali ativo consumido durante o cozimento para determinado número kappa preestabelecido.

O sucesso do emprego desta técnica requer o uso de equipamentos em excelentes condições, estáveis e aferidos e, certamente, de analistas com perfeito treinamento e vasta experiência na execução dos cozimentos. Tais exigências são indispensáveis em estudos de seleção de clones utilizando apenas uma batelada de quatro cozimentos, sem necessidade de repetições. 


\section{REFERÊNCIAS BIBLIOGRÁFICAS}

DOWNES, G.M. et al. Sampling plantation Eucalyptus for wood and fiber properties. Austrália: CSIRO Publishing, 1997. $132 \mathrm{p}$.

FLORES, D.M.M. et al. Amostragem de árvores para estudos tecnológicos da madeira para produção de celulose: tamanho da amostra, número mínimo de repetições e variabilidade das propriedades para clone de Eucalyptus saligna Smith. O Papel, v. 61, n. 3, p. 44 - 55, 2000.
FONSECA, S.M.; OLIVEIRA, R. C.; SILVEIRA, P. N. Seleção da árvore industrial. Revista Árvore, v. 20, n. 01, p. 69-85, 1996.

GRACE, T. M. et al., (Ed.) Kraft liquor. In: Pulp and paper manufacture,

Alkaline pulping. Atlanta: Tappi, 1989. v. 1, p. 15-22.

TECHNICAL ASSOCIATION OF THR PULP AND PAPER INDUSTRY - TAPPI. Test methods. Atlanta: Tappi, 2001-2002. Paginação irregular. 